\title{
NECESIDAD DE TRATAMIENTO ENDODÓNTICO EN ESCOLARES DE SEIS AÑOS EN DOS PARROQUIAS DE LA CIUDAD DE CUENCA 2016
}

\section{NEED FOR ENDODONTIC TREATMENT OF SIX YEARS SCHOOL CHILDREN OF TWO DISTRICTS OF CUENCA CITY 2016}

\author{
Rivera Dahianna ${ }^{1 *}$, Bastidas Zulay ${ }^{2}$ \\ ${ }^{1}$ Estudiante de la Universidad Católica de Cuenca. Ecuador \\ ${ }^{2}$ Especialista en Endodoncia, Catedrática de la Universidad Católica de Cuenca. Ecuador \\ *dtriveral88@est.ucacue.edu.ec
}

\begin{abstract}
Resumen
OBJETIVO: Determinar la frecuencia de necesidad de tratamiento endodóntico en escolares de seis años de edad de las parroquias urbanas Bellavista y Gil Ramírez Dávalos en la ciudad de Cuenca-Ecuador 2016. MATERIALES Y MÉTODOS: Estudio transversal de evaluación clínica bucal en 302 escolares de seis años de edad con dentición decidua en las parroquias urbanas Bellavista y Gil Ramírez Dávalos Cuenca-Ecuador 2016. De esta muestra fueron excluidos los que presentaron enfermedades sistémicas y malformaciones bucales, se utilizaron los criterios de İndice p.u.f.a para la necesidad de tratamiento endodóntico. Los observadores fueron calibrados por un especialista, los padres firmaron el consentimiento y los escolares dieron el asentimiento informado. Las revisiones clínicas intraorales fueron desarrollados antes del recreo, con luz natural y espejo bucal. RESULTADOS: El total de la muestra fue de 302 escolares de las parroquias Bellavista y Gil Ramírez Dávalos, de los cuales el $38 \%$ representa al sexo masculino y $62 \%$ al sexo femenino; $11 \%$ presentaron necesidad de tratamiento endodóntico, $10 \%$ principalmente por pulpitis y $1 \%$ por úlcera. CONCLUSIONES: Este levantamiento de datos revela que existe la necesidad de tratamiento endodóntico a los seis años de edad, con una prevalencia del $11 \%$, que debe ser atendido por el odontopediatra y endodoncista.
\end{abstract}

Palabras clave: Terapia pulpar, odontopediatria, pulpitis, necrosis..

\begin{abstract}
AIM Establish the frequency of requirement for endodontic treatment for six year olds in Bellavista and Gil Ramírez Dávalos urban districts at the city of Cuenca-Ecuador 2016. MATERIALS AND METHODS: Cross-sectional study of oral clinical evaluation of 302 six year-old school children with deciduous dentition in Bellavista and Gil Ramírez. Dávalos urban districts at Cuenca-Ecuador 2016. Out of this sample, those who presented systemic diseases and oral malformations were excluded. Use the diagnostic criteria for the need for endodontic treatment. A specialist calibrated observers, the parents signed the consent, and the students were given informed consent. The clinical intraoral examinations were performed before recess, with natural light and mouth mirror. RESULTS: A total of 302 school children from the Bellavista and Gil Ramírez Dávalos districts, which $38 \%$ represent males and $62 \%$ females; $11 \%$ had the need for endodontic treatment, $10 \%$ mainly for pulpitis and 1\% for ulcer. CONCLUSIONS: This data collection reveals that there is a need for endodontic treatment at six years of age, with a prevalence of $11 \%$, which must be attended by the pediatric dentistry and the endodontist.
\end{abstract}

Key words: Pulpal therapy, pediatric dentistry, pulpitis, necrosis..

\section{Introducción}

La caries dental es una enfermedad mundial, se presenta como la de mayor prevalencia afectando al $90 \%$ de la población; la misma cuando no es tratada, se convierte en un potencial de riesgo para la salud general del paciente y es una urgencia estomatológica. ${ }^{12}$ Según la Organización Mundial de Salud (OMS), las enfermedades bucales son la cuarta causa más incidente, afectando a las poblaciones menos favorecidas. ${ }^{3}$ Para el presente estudio se utilizó el 


\begin{tabular}{lll}
\hline SEXO & TOTAL \\
\hline & $\mathbf{n}$ & $\mathbf{\%}$ \\
Femenino & 188 & 62 \\
Masculino & 114 & 38 \\
Total general & 302 & 100 \\
\hline
\end{tabular}

Tabla 1. Distribución de la muestra de acuerdo al sexo. Fuente: Elaboración propia.

índice p.u.f.a, el mismo que fue introducido por Bella Monse en el año 2006 para valorar el estado de la cavidad oral como resultado de caries no tratadas y establecer cuáles son las consecuencias más frecuentes que pueden ser: compromiso pulpar, úlcera, fístula y absceso. ${ }^{4}$

\section{MATERIALES Y MÉTODOS}

Estudio transversal de evaluación clínica bucal en 302 escolares de 6 años de edad, de las parroquias Bellavista y Gil Ramírez Dávalos Cuenca-Ecuador. Fueron incluidos escolares con dentición decidua y excluidos los que presentaron enfermedades sistémicas y malformaciones bucales, se utilizaron los criterios de índice p.u.f.a para la necesidad de tratamiento endodóntico, teniendo cuatro designaciones: $p$ pulpitis; u úlceras; f fístulas; a abscesos. ${ }^{4}$ Los examinadores fueron calibrados por un especialista, los padres firmaron el consentimiento y los escolares dieron el asentimiento informado.

Todos los exámenes fueron desarrollados antes del recreo, con luz natural y espejo bucal por estudiantes de la Universidad Católica de Cuenca y el análisis estadístico por los mismos.

\section{RESULTADOS}

La muestra total fue de 302 escolares de las parroquias Bellavista y Gil Ramírez Dávalos, de los de los cuales el $38 \%$ representa al sexo masculino y $62 \%$ al sexo femenino (Tabla 1); $11 \%$ presentaron necesidad de tratamiento endodóntico (Gráfico 1), siendo $10 \%$ principalmente por pulpitis y $1 \%$ por úlcera (Tabla 2)

\section{DISCUSIÓN}

En el presente estudio se evidenció una baja prevalencia de condiciones clínicas de caries no tratadas en niños de 6 años de edad, con un predominio en el sexo femenino existiendo la necesidad de tratamiento endodóntico, principalmente por pulpitis y por úlcera.

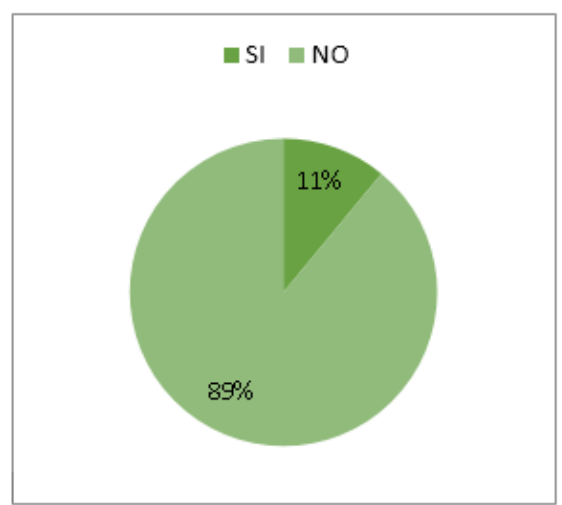

Grf. 1. Necesidad de tratamiento endodóntico. Fuente: Elaboración propia.

\begin{tabular}{lllllll}
\hline $\begin{array}{l}\text { ÍNDICE } \\
\text { p.u.f.a }\end{array}$ & \multicolumn{2}{l}{ FEMENINO } & \multicolumn{2}{l}{ MASCULINO } & $\begin{array}{l}\text { TOTAL } \\
\text { GENERAL }\end{array}$ \\
\hline & $\mathbf{n}$ & $\%$ & $\mathbf{n}$ & $\%$ & $\mathbf{n}$ & $\%$ \\
Pulpitis & 20 & 7 & 9 & 3 & 29 & 10 \\
Úlcera & 2 & 1 & 0 & 0 & 2 & 1 \\
Fístula & 0 & 0 & 1 & 0 & 1 & 0 \\
Absceso & 0 & 0 & 1 & 0 & 1 & 0 \\
$\begin{array}{l}\text { Sano } \\
\begin{array}{l}\text { Total } \\
\text { General }\end{array}\end{array}$ & 166 & 55 & 103 & 34 & 269 & 89 \\
\hline
\end{tabular}

Tabla 2. Distribución de la población de acuerdo al índice p.u.f.a y según el sexo. Fuente: Elaboración propia.

Este tipo de condiciones orales no son solo un problema en Ecuador sino que de la misma forma afecta a diferentes partes de Latino América y del mundo.

Da Silva, 2011 en la ciudad de Paranoá Brasil el índice p.u.f.a. obtenido en su estudio fue $23.7 \%$. $^{5}$

Villavicencio, 2015 analizó en Lima, Perú las lesiones no tratadas, con una prevalencia de $6 \%{ }^{6}$

Grund, 2015 distrito de Westfalia Ennepe-Ruhr- Alemania, desarrolló un estudio donde la prevalencia de infecciones odontogénicas fue $4,4 \%{ }^{7}$

Monse B, 2006 En Filipinas realizó un estudio para evaluar la prevalencia y severidad de las condiciones orales que resultan de las caries dentales sin tratar. La prevalencia fue $85 \%$ en niños de 6 años. ${ }^{4}$

Baginska J. 2013 La prevalencia de las consecuencias clínicas de caries no tratadas fue $40.77 \%$ en el Distrito de Bialystok, Polonia. ${ }^{8}$ 
Rohini Dua 2014, Un estudio en niños de la zona rural de India manifestó que el índice pufa variaba según la condición económica de estos y presentaban entre $2.25-3.00{ }^{9}$

Patil Snehal, 2014 En Western Maharashtra, distrito de la India, la prevalencia de caries no tratadas fue $24 \%$ un valor alto con respecto a nuestro estudio. ${ }^{10}$

Rodakowska E, 2011 en Polonia la prevalencia de las consecuencias clínicas de caries no tratadas en dentición temporal fue $43.4 \% .{ }^{11}$

Existen también estudios a través del índice P.U.F.A. que valoran las consecuencias de caries no tratadas en la dentición permanente.

\section{CONCLUSIÓN}

Este levantamiento de datos revela que existe necesidad de tratamiento endodóntico a los 6 años de edad, con una prevalencia del $11 \%$ que debe ser atendida por el odontopediatra y el endodoncista, con la finalidad de mejor el estado de salud bucal de los escolares.

Conflicto de intereses y financiamiento Los autores declaran no tener conflicto de intereses, haber cumplido con los requisitos de autoría y haber autofinanciado este artículo.

\section{Referencias}

1 Gato I.La caries dental. Algunos de los factores relacionados con su formación en niños.. Revista Cubana de Estomatología.. 2008 Mar; 45(1).

2 Javier. F. Urgencias por caries dental en niños de 4-12 años en Piritú,Venezuela.

3 Vce. R. Caries dental en escolares de 12 años de la parroquía Sayausí (cuenca). Odontología Activa Ucacue. 2016 Ene; 1(1).

4 M B. Un índice de consecuencias clínicas de la caries dental no tratadas. Comunidad Dent Oral Epidemiol. 2010 Feb; 38(1).

5 Mjd S. Prêvalencia de cárie em Escolares do Paranoá/DF. 2011 Dic.

6 PG. A. Prevalencia de Caries de Infancia Temprana. Repositorio Académico USMP. 2015.

7 Katrin Grund A. Clinical consequences of untreated dental caries in German 5- and 8-year-olds. BMC Oral Health.. 2015. Nov.; 15(40).

8 Ws. JB. Pulpal Involvement-Roots-Sepsis Index. Medical Principles and practices.. 2013 Jul.

9 Rjdkna. RD. Correlation between PUFA/pufa.. Department of Pedodontic and Preventive Dentistry. 2016 Jul.

10 Ssshsrsask. PS. Prevalence of untreated dental caries among the preschool children of Western Maharashtra.. Department of Public Health Dentistry. 2015. Sep.

11 Rewj. BJ. Index of clinical consequences of untreated dental.. Medical University of Bialystok, Poland. 2012 Dic.; $58((2))$. 
